\title{
Frequency of Genital Infections According to Body Mass Index in Dapagliflozin-treated Patients with Type 2 Diabetes Mellitus
}

\section{() (1) $\odot \Theta$}

\section{Authors}

Gottfried Rudofsky ${ }^{1}$, Tanja Haenni ${ }^{1}$, John Xu², Eva Johnsson ${ }^{3}$

Affiliations

1 Department of Endocrinology, Diabetes and Clinical Nutrition, Kantonsspital, Olten, Switzerland

2 AstraZeneca, Gaithersburg, MD, USA

3 AstraZeneca, Gothenburg, Mölndal, Sweden

Key words

sodium glucose co-transporter 2 inhibitors, obesity, counselling

received 25.08.2016

revised 17.11 .2016

accepted 02.12.2016

\section{Bibliography}

DOI http://dx.doi.org/10.1055/s-0043-101823

Published online: March 16, 2017

Exp Clin Endocrinol Diabetes Rep 2017; 5: e1-e4

(c) J. A. Barth Verlag in Georg Thieme Verlag KG Stuttgart - New York ISSN 2196-7407

\author{
Correspondence \\ Gottfried Rudofsky, MD \\ Kantonsspital Olten \\ Baslerstr. 150 \\ 4600 Olten \\ Switzerland \\ Tel.: + 49/62/31142 37, Fax: + 49/62/3114124 \\ Gottfried.rudofsky@spital.so.ch
}

\begin{abstract}
Aims Genital infections are associated with sodium glucose cotransporter 2 inhibitors such as dapagliflozin. Since patients with Type 2 diabetes are at increased risk of genital infections, and obesity is a risk factor for infections, obese patients with Type 2 diabetes could be more susceptible to genital infections when treated with sodium glucose co-transporter 2 inhibitors. This pooled dataset assessed the frequency of genital infections according to baseline body mass index in patients treated with dapagliflozin $10 \mathrm{mg}$.

Methods Data were pooled from 13 studies of up to 24 weeks' duration (dapagliflozin $N=2360$; placebo $N=2295$ ). Frequency of genital infections was compared between three body mass index subgroups $\left(<30, \geq 30-<35\right.$ and $\left.\geq 35 \mathrm{~kg} / \mathrm{m}^{2}\right)$.

Results Genital infections were reported in 130 (5.5\%) patients receiving dapagliflozin and 14 (0.6\%) patients receiving placebo; none of which were serious. Genital infections were more common in women $(84 / 130$ [64.6\%]) than in men (46/130 [35.4\%]) treated with dapagliflozin. In the body mass index $<30, \geq 30-<35$ and $\geq 35 \mathrm{~kg} / \mathrm{m}^{2}$ dapagliflozin-treated subgroups, 38/882 (4.3\%), 47/796 (5.9\%) and 45/682 (6.6\%) patients presented with genital infections, respectively. Conclusion Although the frequency was low overall and relatively similar between subgroups, there was a trend towards an increase in genital infections in patients with a higher body mass index. This trend is unlikely to be clinically relevant or to affect suitability of dapagliflozin as a treatment option for obese patients with Type 2 diabetes, but rather should influence advice and counselling of overweight patients on prevention and treatment of genital infections.
\end{abstract}

\section{Introduction}

Treatment with sodium glucose co-transporter 2 (SGLT2) inhibitors is associated with an increased incidence of genital infections, such as vulvovaginitis, balanitis and other related genital infections; however, in clinical studies, the overall frequency of these events is generally low $(\sim 5 \%)[1,2]$. Furthermore, data from these studies show that genital infections were more common in women than in men, and were mostly mild or moderate in intensity [2].

In addition to female gender being a risk factor for genital infections, studies have shown that Type 2 diabetes mellitus (T2DM) and a history of urinary tract infections (UTIs) or genital infections (from organisms such as E. coli, Klebsiella sp and Proteus sp and C. albicans, which typically flourish in the hyperglycaemic environment of T2DM) can predispose to further UTIs and genital infections [1]. This trend was observed irrespective of whether patients were receiving SGLT2 inhibitor treatment or placebo [1]. However, because obesity is a potential risk factor for some infections [3], and the anatomy and physicality of obese patients may predispose them to genital infections due to increased skin folds and perspiration creating a hospitable environment for bacterial and mycotic growth, it could be speculated that people with a higher body weight receiving SGLT2 inhibitors are at increased risk of genital infections.

Dapagliflozin is a highly selective SGLT2 inhibitor that improves glycaemic control in people with T2DM by inhibiting renal glucose reabsorption, resulting in increased glucosuria [4]. We conducted a pooled analysis to assess the frequency of genital infections according to baseline body mass index (BMI) in people with T2DM treated with dapagliflozin, to determine whether overweight people have a clinically meaningful increased susceptibility to this adverse event. 
- Table 1 Demographic and baseline characteristics *

\begin{tabular}{|c|c|c|c|c|}
\hline & \multicolumn{2}{|c|}{ No genital infection } & \multicolumn{2}{|c|}{$\geq 1$ genital infection } \\
\hline & $\begin{array}{c}\text { Placebo } \\
(N=2281)\end{array}$ & $\begin{array}{l}\text { DAPA } 10 \mathrm{mg} \\
(N=2227)\end{array}$ & $\begin{array}{l}\text { Placebo } \\
(N=14)\end{array}$ & $\begin{array}{c}\text { DAPA } 10 \mathrm{mg} \\
(N=130)\end{array}$ \\
\hline Age, years & $59.0(9.9)$ & $58.4(10.0)$ & $51.1(14.4)$ & $57.8(10.2)$ \\
\hline \multicolumn{5}{|l|}{ Gender, $n(\%)$} \\
\hline Male & $1340(58.7)$ & $1309(58.8)$ & $3(21.4)$ & $46(35.4)$ \\
\hline Female & $941(41.3)$ & $918(41.2)$ & $11(78.6)$ & $84(64.6)$ \\
\hline \multicolumn{5}{|l|}{ Race, $n(\%)$} \\
\hline White & $1919(84.1)$ & $1862(83.6)$ & $11(78.6)$ & $111(85.4)$ \\
\hline Black or African American & $71(3.1)$ & $72(3.2)$ & $2(14.3)$ & $9(6.9)$ \\
\hline Asian & $205(9.0)$ & $203(9.1)$ & $1(7.1)$ & $6(4.6)$ \\
\hline Other & $86(3.8)$ & $90(4.0)$ & 0 & $4(3.1)$ \\
\hline Weight, kg & $90.2(19.3)$ & $90.4(19.8)$ & $84.4(20.6)$ & $92.3(18.4)$ \\
\hline \multicolumn{5}{|l|}{ BMI categorization, $n$ (\%) } \\
\hline$<30 \mathrm{~kg} / \mathrm{m}^{2}$ & $876(38.4)$ & $844(37.9)$ & $9(64.3)$ & $38(29.2)$ \\
\hline $30-35 \mathrm{~kg} / \mathrm{m}^{2}$ & $766(33.6)$ & $748(33.6)$ & $1(7.1)$ & $47(36.2)$ \\
\hline$\geq 35 \mathrm{~kg} / \mathrm{m}^{2}$ & $639(28.0)$ & $635(28.5)$ & $4(28.6)$ & $45(34.6)$ \\
\hline \multicolumn{5}{|l|}{ HbA1c } \\
\hline $\mathrm{mmol} / \mathrm{mol}$ & $66(10)$ & $66(10)$ & $70(11)$ & $65(9)$ \\
\hline$\%$ & $8.2(0.9)$ & $8.2(1.0)$ & $8.6(1.0)$ & $8.1(0.8)$ \\
\hline Diabetes duration, years & $8.8(8.0)$ & $8.8(8.0)$ & $8.6(6.2)$ & $10.2(8.4)$ \\
\hline
\end{tabular}

\section{Patients and Methods}

Data were pooled from 13 phase 2b/3 studies (12-24 weeks' duration) assessing treatment with dapagliflozin $10 \mathrm{mg}$ (as monotherapy, initial combination with metformin, add-on therapy to oral antidiabetic drugs [OADs] or as add-on therapy to OADs plus insulin) vs. placebo (Supplementary $>$ Table S1).

In the analysed studies, adverse events of genital infection were obtained via proactive questioning at study visits and via spontaneous reporting, using a pre-defined list of preferred terms from the Medical Dictionary for Regulatory Activities (MedDRA ${ }^{\circledR}$; version 15.1) (Supplementary $\triangleright$ Table S2).

\section{Outcome measures}

The frequency of genital infections over 24 weeks was evaluated according to baseline BMI for the following subgroups: Subgroup 1: $\mathrm{BMI}<30 \mathrm{~kg} / \mathrm{m}^{2}$; Subgroup 2: BMI $\geq 30$ to $<35 \mathrm{~kg} / \mathrm{m}^{2}$; Subgroup $3: \mathrm{BMI} \geq 35 \mathrm{~kg} / \mathrm{m}^{2}$. Each BMI category was further subdivided into men and women.

\section{Statistical analyses}

The odds ratio of genital infections in the BMI subgroup $\geq 35 \mathrm{~kg} / \mathrm{m}^{2}$ vs. $<30 \mathrm{~kg} / \mathrm{m}^{2}$ was calculated. The rate of patients with a genital infection was estimated using Kaplan-Meier curves based on timeto-first genital infection. Comparison of dapagliflozin-treated patients with genital infections between the different BMI subgroups was performed using $P$-values calculated by log-rank test. Adverse events data were summarized using descriptive statistics.

\section{Results}

In total, 4655 patients were included in this pooled analysis: 2360 patients treated with dapagliflozin $10 \mathrm{mg}$ and 2295 patients treated with placebo. Three of the 2360 patients who received dapagliflozin were not randomized to this treatment arm and are therefore not included in the baseline and demographics table. Overall, 882, 796 and 682 dapagliflozin-treated patients were included in the $<30, \geq 30$ to $<35$ and $\geq 35 \mathrm{~kg} / \mathrm{m}^{2}$ BMl subgroups.

Demographic and baseline characteristics for the dapagliflozinand placebo-treated groups with and without a genital infection are reported in $>$ Table 1 . Adverse events of genital infection were reported in $130(5.5 \%)$ and $14(0.6 \%)$ patients receiving dapagliflozin and placebo, respectively, whereas no events of genital infection were reported in 2227 (94.5\%) patients receiving dapagliflozin and 2281 (99.4\%) patients receiving placebo. No serious adverse events of genital infection were reported in either group.

Baseline mean $\mathrm{HbA} 1 \mathrm{c}$ was similar in dapagliflozin-treated patients with and without a genital infection $(65 \mathrm{mmol} / \mathrm{mol}[8.1 \%]$ and $66 \mathrm{mmol} / \mathrm{mol}$ [8.2\%], respectively) ( $\triangleright$ Table 1 ). The mean age (standard deviation [SD]) of dapagliflozin-treated patients with and without genital infections was 57.8 (10.2) and 58.4 (10.0) years, respectively, and the mean duration of diabetes (SD) was 10.2 (8.4) and 8.8 (8.0) years, respectively. Adverse events of genital infection were more common in women (64.6 and 78.6\%) than in men (35.4 and 21.4\%) treated with dapagliflozin or placebo, respectively ( $\vee$ Table 1 ).

Adverse events of genital infection, analysed according to BMI category, were reported in 38 (4.3\%) patients receiving dapagliflozin 


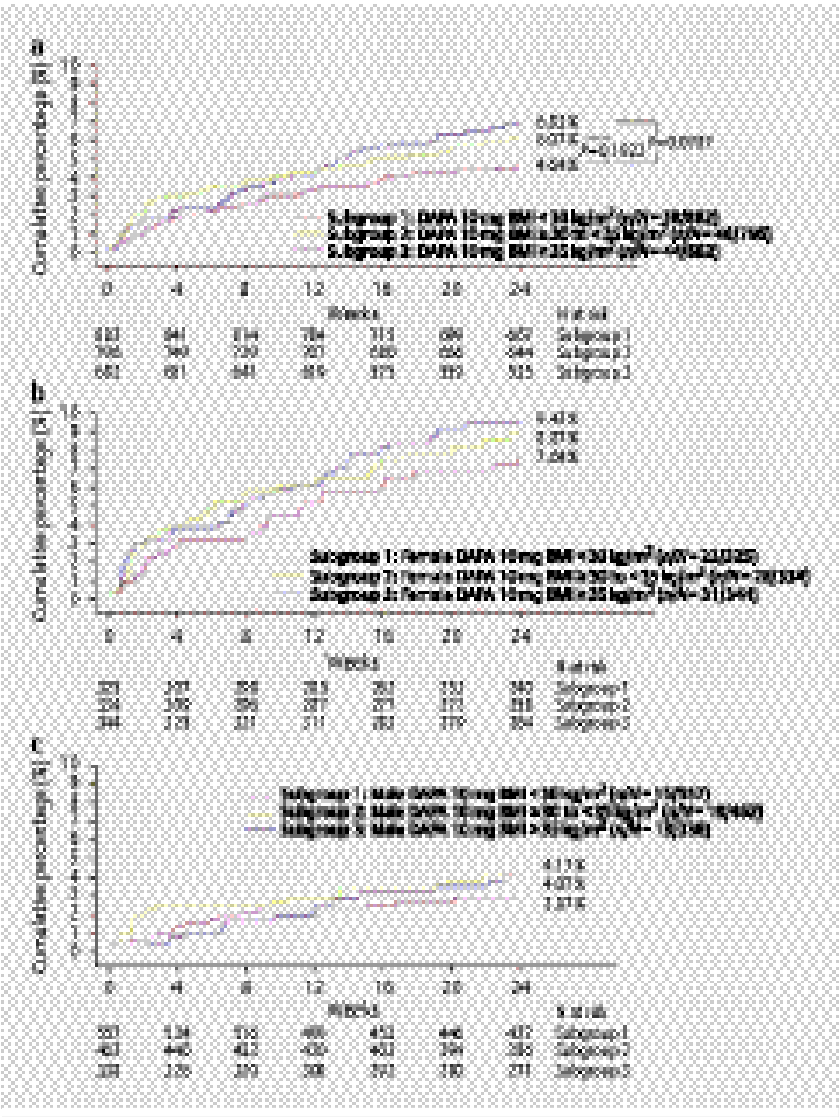

- Fig. 1 Kaplan-Meier estimates of the proportion of dapagliflozin-treated a total number of patients, $\mathbf{b}$ women and $\mathbf{c}$ men with adverse events of genital infection over 24 weeks. Data shows time-to-first infection. Values are based on estimated events. $n=$ number of patients with any adverse event of genital infection; $N=$ total number of patients in the subgroup. $P$-values were calculated by log-rank test. BMI, body mass index; DAPA, dapagliflozin.

in the $\mathrm{BMI}<30 \mathrm{~kg} / \mathrm{m}^{2}$ category, in 47 (5.9\%) patients in the $\mathrm{BMI} \geq 30$ to $<35 \mathrm{~kg} / \mathrm{m}^{2}$ category and in $45(6.6 \%)$ patients in the $\mathrm{BMI} \geq 35 \mathrm{~kg} /$ $\mathrm{m}^{2}$ category. The most common adverse events of genital infection in the dapagliflozin group for all three BMI categories were vulvovaginal mycotic infections, vaginal infections and balanitis (Supplementary $>$ Table S3). There were few discontinuations due to genital infections reported in the dapagliflozin treatment group $(n=5)$ and the number was similar across BMI subgroups: 2 patients in the $\mathrm{BMI}<30 \mathrm{~kg} / \mathrm{m}^{2}$ group, one patient in the BMI $\geq 30$ to $<3 \mathrm{~kg} / \mathrm{m}^{2}$ group and 2 patients in the $\mathrm{BMI} \geq 35 \mathrm{~kg} / \mathrm{m}^{2}$ group.

Kaplan-Meier estimates reporting time-to-first infection showed that the rate of genital infections with dapagliflozin was higher in the first few weeks ( $~ 4$ weeks) of the treatment period in the overall population ( $\vee$ Fig. 1a) and in women ( $\mathbf{F i g}$. 1b). Furthermore, genital infections in women appeared to be more BMI-dependent compared with men ( $\mathbf{F i g} \mathbf{1} \mathbf{1 b}, \mathbf{c})$.

Although there were no statistically significant differences between BMI groups according to the Kaplan-Meier analysis ( $\mathbf{F i g . ~ 1 a ) , ~}$ comparison of the odds ratio for genital infections in the dapagliflozin $10 \mathrm{mg}$ BMI subgroup $\geq 35 \mathrm{~kg} / \mathrm{m}^{2}$ with the dapagliflozin $10 \mathrm{mg}$ BMI subgroup $<30 \mathrm{~kg} / \mathrm{m}^{2}$ was 1.57 , indicating that the like- lihood of genital infections was $57 \%$ greater in patients with a BMI of $\geq 35 \mathrm{~kg} / \mathrm{m}^{2}$ compared with a BMI of $<30 \mathrm{~kg} / \mathrm{m}^{2}$.

\section{Discussion}

This pooled analysis assessed the frequency of genital infections according to baseline BMI in dapagliflozin-treated people with T2DM. Overall, the frequency of adverse events of genital infection was low in people treated with dapagliflozin $10 \mathrm{mg}$. A trend towards a greater frequency of genital infections was observed in people with a higher baseline BMI, with the odds of genital infections in the dapagliflozin $10 \mathrm{mg}$ group increasing by $57 \%$ in people with a BMI of $\geq 35 \mathrm{~kg} / \mathrm{m}^{2} \mathrm{vs}$. a BMI of $<30 \mathrm{~kg} / \mathrm{m}^{2}$.

However, despite the observation of a trend towards greater frequency of genital infections in people with a higher baseline BMI, this is unlikely to influence the decision whether to use the drug in overweight people, due firstly to the relatively low frequencies of genital infections (ranging from 4.3-6.6\% in the different BMI categories) in this analysis, and due secondly to the benefits of SGLT2 inhibitor treatment in this population. Specifically, dapagliflozin has been shown to improve glycaemic control, in addition to reducing body weight, blood pressure and serum uric acid $[4,5]$, all factors which contribute to complications and comorbidities in people with obesity [6]. In addition, the findings from the recent EMPA-REG OUTCOME ${ }^{\circledR}$ trial in patients with T2DM and established cardiovascular disease [7] have demonstrated the beneficial effects of this SGLT2 inhibitor treatment on cardiovascular events and mortality.

Based on these considerations, we believe that there is no need to adjust treatment in overweight people receiving SGLT2 inhibitor treatment. However, physicians should be aware of this potential risk of increased genital infections with increasing BMI, and should consider educating, monitoring and advising overweight patients as appropriate regarding awareness, prevention and treatment of genital infections [8].

Limitations of this study include a low number of genital infection events reported, which made comparisons between groups difficult to interpret. In addition, the duration of the analysis (12-24 weeks) only enabled short-term assessment of adverse events of genital infection. Finally, since a BMI of $>45 \mathrm{~kg} / \mathrm{m}^{2}$ was an exclusion criterion in the original studies, no conclusions can be made regarding dapagliflozin treatment in people with severe obesity.

In summary, in patients with T2DM treated with dapagliflozin $10 \mathrm{mg}$ the frequency of genital infections was low, irrespective of BMI subgroup. Although these findings were hypothesis-generating only, there was a trend towards a greater frequency of genital infections in patients with a higher baseline BMI. While this trend is unlikely to be clinically relevant or to affect prescription of dapagliflozin for overweight patients with T2DM, physicians may want to raise awareness and provide counselling on the prevention and treatment of genital infections.

\section{Acknowledgements}

Gottfried Rudofsky was supported by a grant from the European Foundation of Studies in Diabetes (Clinical Research Programme 
2013). The authors would like to thank Irina Baldycheva and Valerie Cain for statistical support. Medical writing support was provided by Maria Thadani-Mulero of inScience Communications, Springer Healthcare, funded by AstraZeneca.

Funding sources: This study was funded by AstraZeneca who was also responsible for the study design, data collection and analysis, and manuscript preparation. The corresponding author had full access to the data in the study and final responsibility for the decision to submit for publication.

\section{Conflict of interest}

GR has received consulting fees from Amgen, AstraZeneca, Eli Lilly, Novo Nordisk, Sanofi Aventis and grant support from the European Foundation of Studies in Diabetes. TH does not have any financial disclosures to declare. JX and EJ are employees and shareholders of AstraZeneca.

One sentence summary: There is a trend towards an increase in genital infections in patients with a higher BMI; although this trend is unlikely to be clinically relevant, physicians may want to raise awareness and provide counselling on the prevention and treatment of genital infections

\section{References}

[1] Geerlings S, Fonseca V, Castro-Diaz D et al. Genital and urinary tract infections in diabetes: impact of pharmacologically-induced glucosuria. Diabetes Res Clin Pract 2014; 103: 373-381

[2] Johnsson KM, Ptaszynska A, Schmitz B et al. Vulvovaginitis and balanitis in patients with diabetes treated with dapagliflozin. J Diabetes Complications 2013; 27: 479-484

[3] Huttunen R, Syrjanen J. Obesity and the risk and outcome of infection. Int J Obes (Lond) 2013; 37: 333-340

[4] Bailey C], Gross JL, Pieters A et al. Effect of dapagliflozin in patients with type 2 diabetes who have inadequate glycaemic control with metformin: a randomised, double-blind, placebo-controlled trial. Lancet 2010; 375: 2223-2233

[5] Weber MA, Mansfield TA, Cain VA et al. Blood pressure and glycaemic effects of dapagliflozin versus placebo in patients with type 2 diabetes on combination antihypertensive therapy: a randomised, double-blind, placebo-controlled, phase 3 study. Lancet Diabetes Endocrinol 2016; 4: $211-220$

[6] Cirillo P, Sato W, Reungjui S et al. Uric acid, the metabolic syndrome, and renal disease. J Am Soc Nephrol 2006; 17: S165-S168

[7] Zinman B, Wanner C, Lachin JM et al. Empagliflozin, cardiovascular outcomes, and mortality in Type 2 diabetes. N Engl J Med 2015; 373 : 2117-2128

[8] Arakaki RF. Sodium-glucose cotransporter-2 inhibitors and genital and urinary tract infections in type 2 diabetes. Postgrad Med 2016; 128: 409-417 